\title{
A Quantitative Mechanism Based on Environmental Compensation for Trash Incineration
}

\author{
Zhenshi Zhang \\ College of Basic Education, National University of \\ Defense Technology, Changsha, China \\ Corresponding author: zhangzhenshi@nudt.edu.cn \\ Huangke Chen \\ College of Information System and Management, \\ National University of Defense Technology, Changsha, \\ China \\ chenhuangke@nudt.edu.cn
}

\begin{abstract}
A trash incineration would pollute the around environment, and the citizen lived there should get compensation. In this paper, we design a pollution inspection model based on the Gaussian Diffusion Model and weather conditions. With the help of this model, the gas concentration distribution around the trash incineration is simulated, and then a quantitative mechanism is proposed to compensate the citizens lived around the trash incineration. We take a middlesized trash incineration in Shenzhen City as an experimental case to demonstrate the solution of our model and an example mechanism.
\end{abstract}

Keywords-Gaussian diffusion model; Gas concentration distribution; Compesation mechanism.

\section{INTRODUCTION}

Trash problem is always a world-wide governmentfocused problem. Because the government lacks efficient management and the investigators ignore the future development, trash incinerations have brought lots of pollution in many cities [1]. In this situation, it is very difficult for newly emerged trash incineration technologies to be popularized [2]. It is more difficult for government to build trash incineration because no citizen like a trash incineration built near their home. For the existing trash incinerations, their manager only focus on the inspection inside the trash incineration. However, the inspection outside the incineration which should be based on environment has not been taken care of enough. Therefore, citizens near the trash incineration are not satisfied. They want government or trash incineration manager to publish a convincible environment inspection system that shows how is the pollution. They want to be compensated, since their environment is polluted $[3,4]$.

In this scenario, this paper designs a dynamic pollution inspection model based on the Gaussian Diffusion Model. Then a quantitative mechanism based on environmental compensation is proposed for citizens near trash incinerations.

\author{
Huanle Pei \\ College of Basic Education, National University of \\ Defense Technology, Changsha, China \\ huanlepei@nudt.edu.cn \\ Yipeng Li \\ Research Department National University of Defense \\ Technology, Changsha, China \\ yipengli@nudt.edu.cn
}

\section{POLLUTION INSPECTION MODEL}

In order to inspect pollution around the trash incineration, we need to calculate the gas concentration in the air. In this section, we use wind direction and speed as input parameters to design a pollution inspection model based on classic Gaussian Diffusion Model [5].

Figure 1 shows the coordinate system of Gaussian Diffusion Model. The original point $O$ is the point or the projection point where gas leaks out, for example the chimney of trash incineration. The positive direction of $\mathrm{X}$ axis is the wind direction. The $\mathrm{Y}$-axis is perpendicular to $\mathrm{X}$ axis, and the Z-axis is perpendicular to the plain $X O Y$ [6].

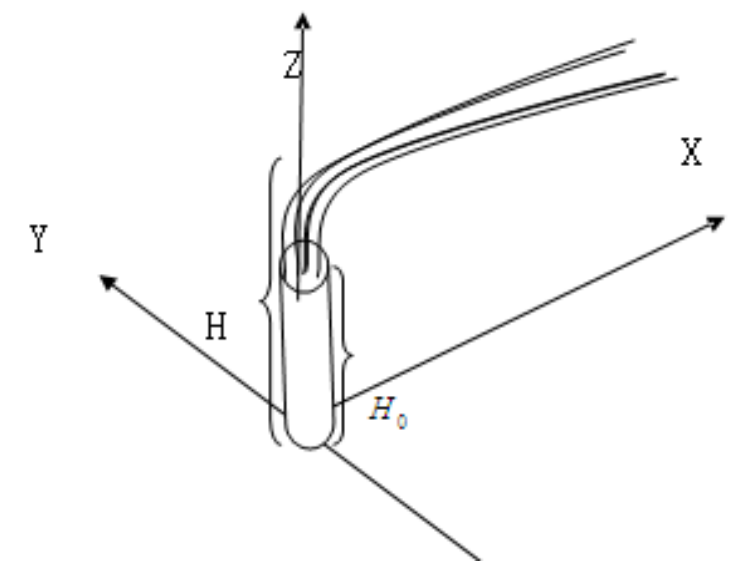

Figure 1. Coordinate system of Gaussian Diffusion Model

Based on the Normal Distribution, the equation of gas concentration is

$$
X(x, y, z)=A(x) e^{-a y^{2}} e^{-b z^{2}}
$$

Then the equation of variance is 


$$
\left\{\begin{array}{l}
\sigma_{y}^{2}=\frac{\int_{0}^{\infty} y^{2} X d y}{\int_{0}^{\infty} X d y} \\
\sigma_{z}^{2}=\frac{\int_{0}^{\infty} z^{2} X d z}{\int_{0}^{\infty} X d z}
\end{array}\right.
$$

The equation of gas integration is

$$
Q=\int_{-\infty}^{\infty} \int_{-\infty}^{\infty} u X d y d z
$$

Where $\sigma_{y}$ is the standard deviation of gas diffusion in Y-axis, and $\sigma_{z}$ is the one in z-axis, and the unit is meter. $X$ indicates the gas concentration, and the unit is kilogram per cube meter. $Q$ indicates the gas leaking speed, and the unit is kilogram per second.

Put Equation (1) into Equation (2),

$$
\left\{\begin{array}{l}
a=\frac{1}{2 \sigma_{y}^{2}} \\
b=\frac{1}{2 \sigma_{z}^{2}}
\end{array}\right.
$$

Put Equation(1) and Equation(4) into Equation(3),

$$
A(x)=\frac{Q}{2 \pi u \sigma_{y} \sigma_{z}}
$$

Put Equation(4) and Equation(5) into Equation(1),

$$
X(x, y, z)=\frac{Q}{2 \pi u \sigma_{y} \sigma_{z}} \exp \left[-\left(\frac{y^{2}}{2 \sigma_{y}^{2}}+\frac{z^{2}}{2 \sigma_{z}^{2}}\right)\right]
$$

In the practical situation, what we concern is the gas concentration on the ground. Therefore, let $z$ be 0 , and then we get,

$$
X(x, y, 0, H)=\frac{Q}{\pi u \sigma_{y} \sigma_{z}} \exp \left[-\frac{1}{2}\left(\frac{y}{\sigma_{y}}\right)^{2}\right] \exp \left(\frac{H^{2}}{2 \sigma_{z}^{2}}\right)
$$

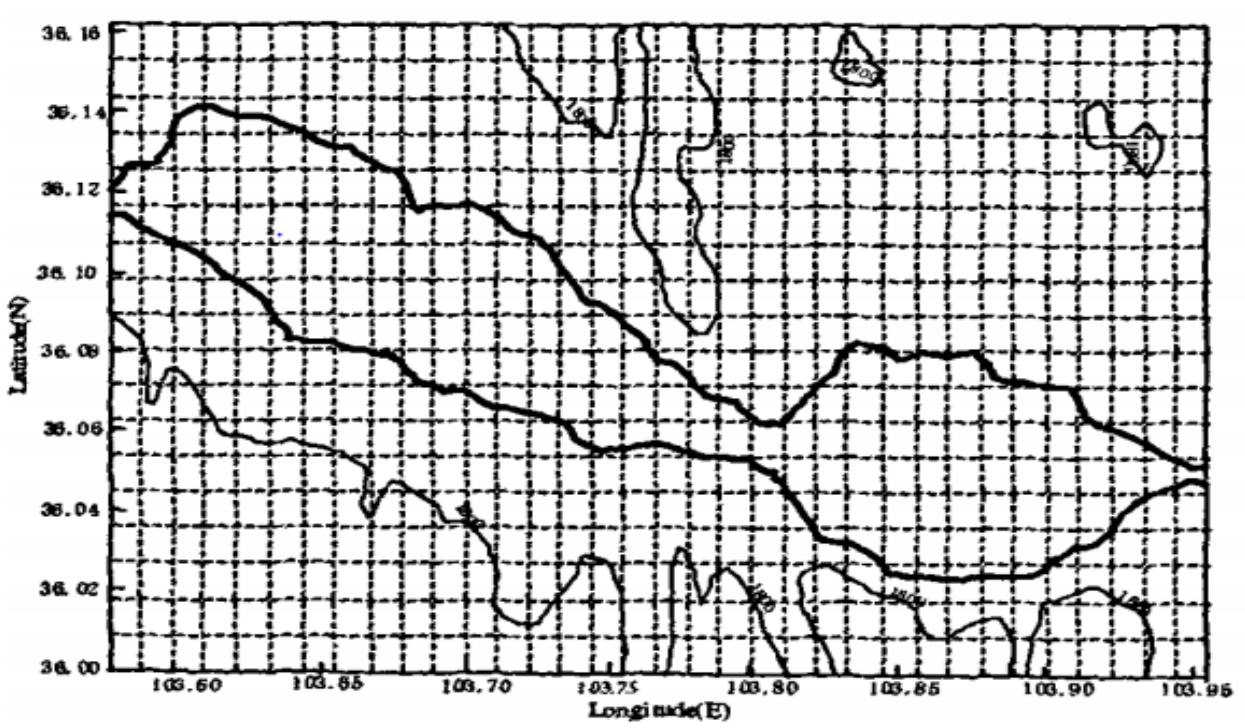

Figure 2. Grid partition of the givin region 


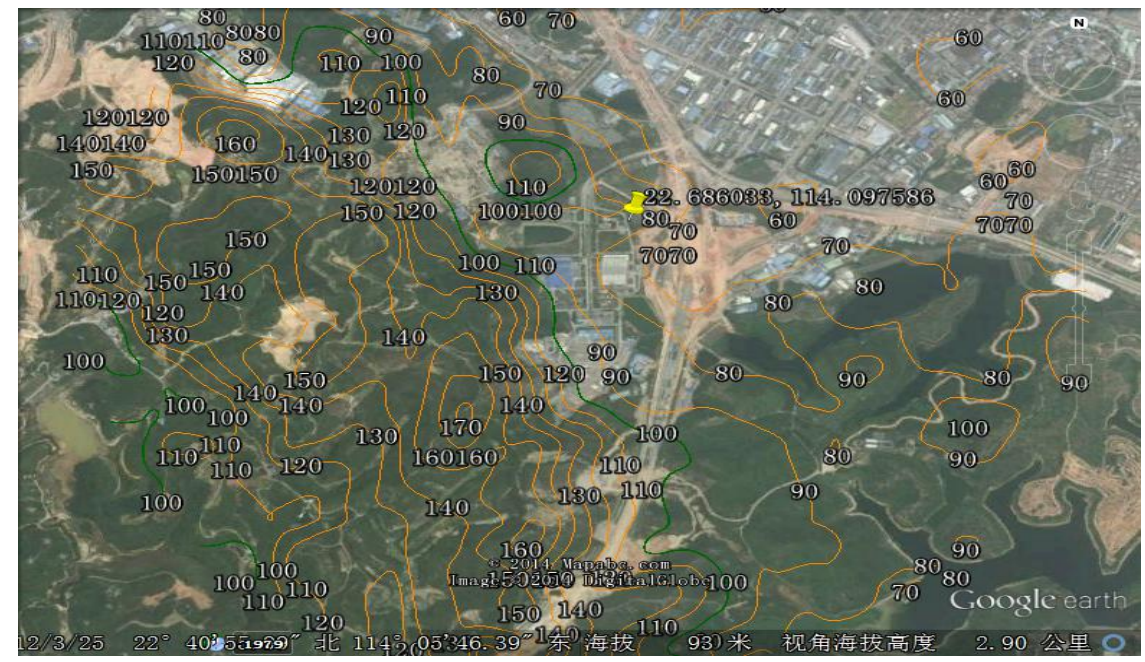

Figure 3. The environmental condition around the trash incineration

To solve Equation (7), there are four steps.

(1) Grid partition

The first step is to partition the region into a grid. Each cell of the grid is a output of the model calculation. The matrix of the grid is the result of pollution, shown in Figure 2.

(2) Parameter calculation

The second step is to calculate the parameters of gas diffusion based on Gaussian Diffusion Model.

(3) Weather prediction

The third step is to predict the weather condition such as wind direction and wind speed via weather prediction government.
(4) Pollution calculation

The last step is to calculate the result of pollution by inputting all of the abovementioned data.

\section{MECHANISM BASED ON ENVIRONMENTAL COMPENSATION}

The main idea of the mechanism is to analyze the compensation region and cost based on the gas concentration we calculate above. Then a reasonable mechanism is proposed to compensate the citizen near the trash incineration.

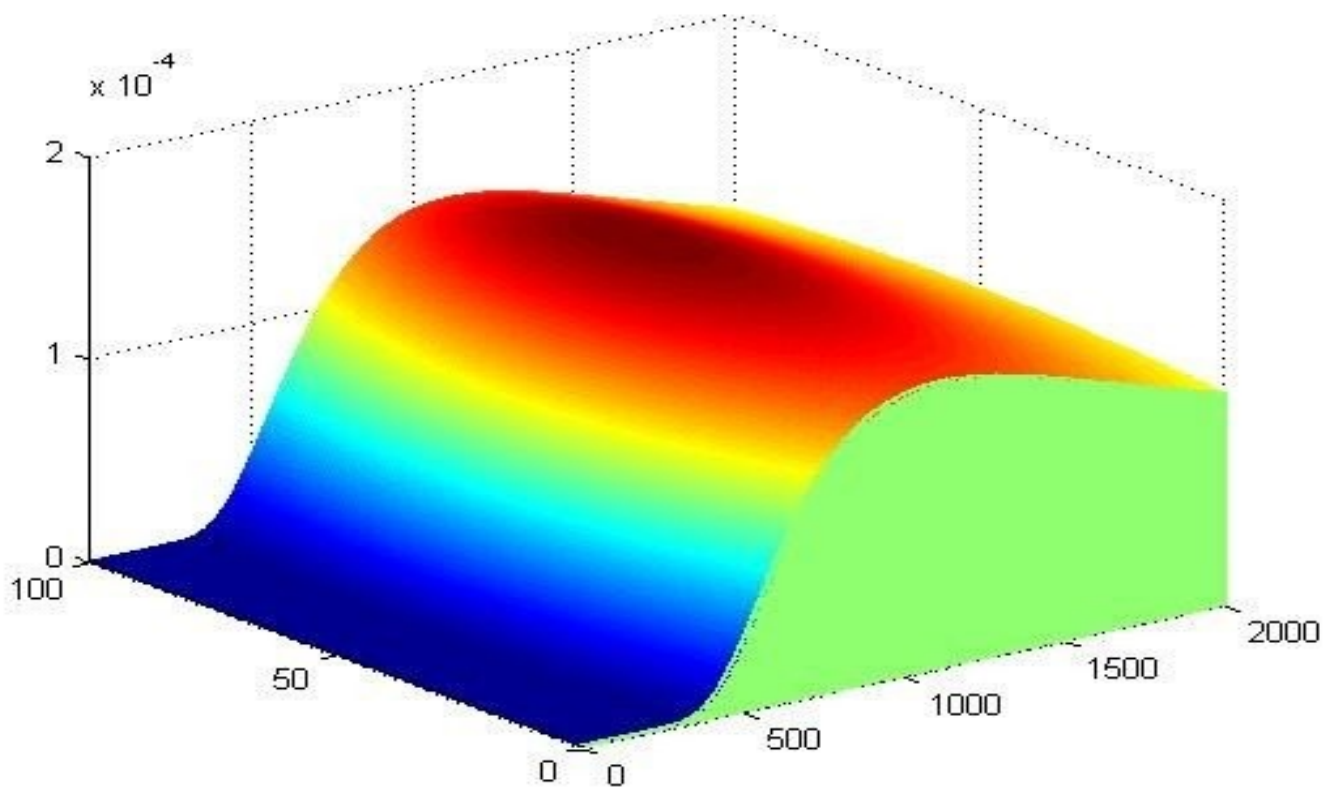

Figure 4. The distribution of gas concentration 


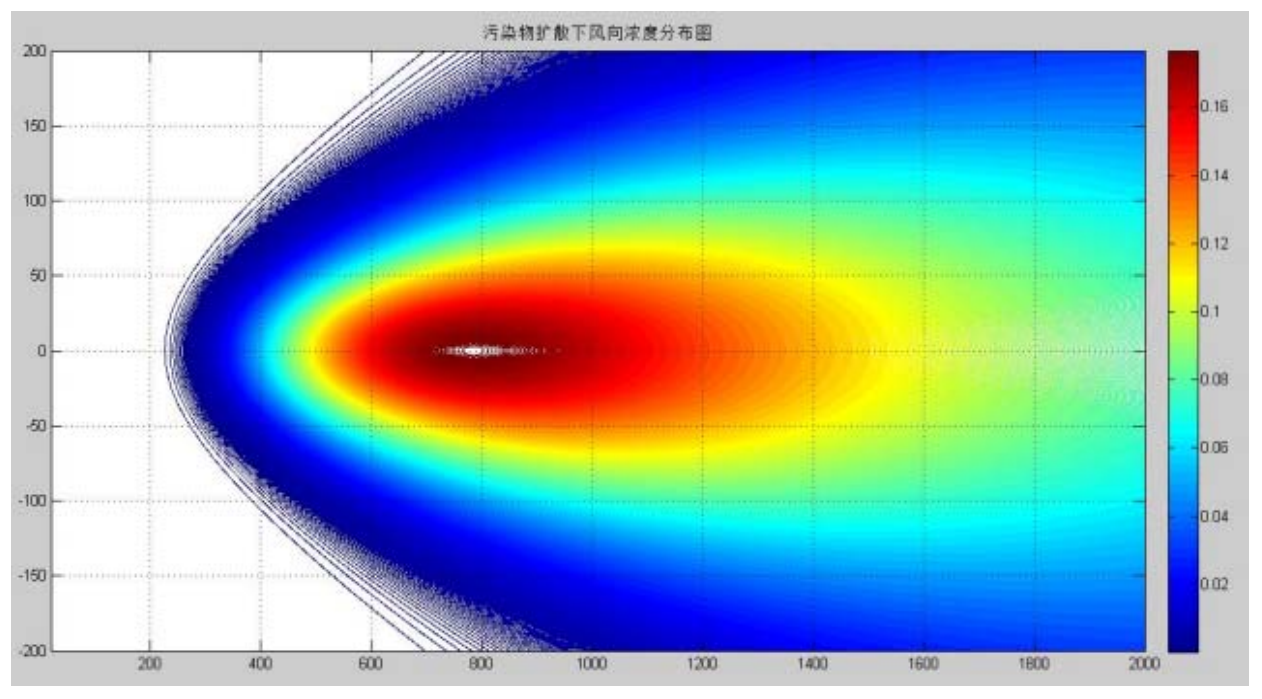

Figure 5. The downwind distribution of gas diffusion

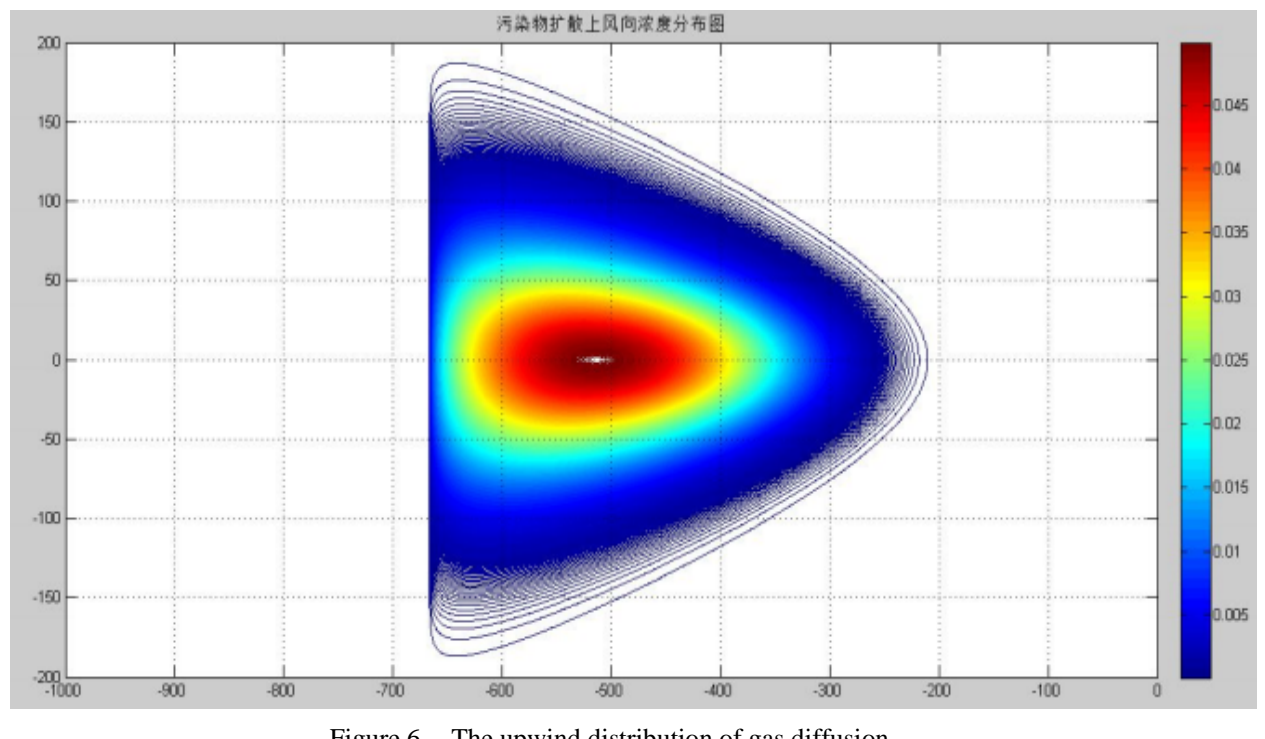

Figure 6. The upwind distribution of gas diffusion

In this paper, we take Shenzhen City as an example to study the mechanism based on environmental compensation. Hypothetically there is a middle-sized trash incineration which processes 1950 tons trash by three incineration machines. Each incineration machine can process 650 tons trash per day. And it is 24-hours working. The height of the chimney is 80 meters.

Firstly, the environmental condition around the trash incineration is analyzed. Figure 3 shows that the region is a flatlands. The elevation is normally low. And the settlement places are all centralized. Because the relief amplitude is not considerable, the model we designed above is suitable for pollution calculation of this region.

Secondly, we use MATLAB to solve the pollution inspection model. The simulation results are shown below.

Figure 4 shows the distribution of gas concentration. From the simulation result we will find the max pollution emerges when the distance to the original point is from 700 meters to 1000 meters. The pollution declines when the distance to the original point is longer than 1500 meters. Therefore, the citizen lived in the region where the distance to the incineration is from 700 meters to 1500 meters should get compensation.

Next, we take the wind condition into our consideration. Figure 5 and Figure 6 show the downwind and upwind distributions of gas diffusion, respectively. It is obvious that the region in the downwind of the trash incineration has more pollution than the one in the upwind. Therefore, the citizen lived downwind should get more compensation than the one lived upwind.

\section{CONCLUSION}

In this paper, we design a model based on Gaussian Diffusion Model to calculate the gas pollution around the 
trash incineration, and then propose a quantitative mechanism based on the pollution distribution. In the future, more conditions should be taken into consideration to improve the model. For example, the gas could be absorbed by rain, but the river could be polluted.

\section{ACKNOWLEDGMENT}

The research in this paper is supported by the Education Program of Hunan Province in China (No.XJK014CCF001).

\section{REFERENCES}

[1] Lightowlers Philip, The dash for trash: UK's incinerator revolution, Environmental Data Services, vol.453, pp.30-33, 2012.
[2] C. Block, J. Van Caneghem, A. Van Brecht, G. Wauters, C. Vandecasteele, Incineration of Hazardous Waste: A Sustainable Process? Waste and Biomass Valorization, vol.6(2), pp.137-145, 2015.

[3] Michael G. Faure, Liu Jing, Compensation for Environmental Damage in China: Theory and Practice, PELR, Vol.31(1), 2014.

[4] Sang Yan-hong,Wu Renhai, Discussion on environmental compensation system, Geographic Environment Research, vol.14(1), pp.50-53, 2002

[5] Wang Weiping, Gaussian diffusion model of the application [J], Zhejiang Meteorological Science and Technology,vol.8, pp.37-45, 2006

[6] Soojin Kang, Se-Jin Yook, Kwan-Soo Lee, Gaussian diffusion sphere model to predict deposition velocities under the combined effects of electrophoresis and thermophoresis, Journal of the Korean Physical Society, vol.64(6), pp.832-839, 2014. 